along the grain boundaries or tiny cracks of the ice toward the centre of the sample.

The phenomenon is not believed to be related to the electrical effects observed during the freezing of aqueous solutions. Certainly no significant changes in the potentials recorded accompanied the addition of the grain of silver nitrate or the subsequent events. The phenomenon does seem of interest, however, in the study of freezing processes and the structure of ice.

The work was done and is continuing under the sponsorship of the U.S. Army Signal Corps.

S. E. REYNoLDS

E. J. WORKMAN

Research and Development Division,

New Mexico School of Mines,

Albuquerque, New Mexico. March 4.

' Workman, E. J., and Reynolds, S. E., Phys, Rev., 74, 709 (1948).

\section{Radiation and Sensation of Pain}

Ir is generally supposed that the only significant reaction produced by absorption, in the tissues, of infra-red radiation-and it applies practically to the radiation from electric lamps - is to cause a rise in temperature of the tissues, there being no attendant chemical effect such as accompanies ultra-violet irradiation. Thus, whereas for ultra-violet irradiation the energy absorption in a single treatment is of the order of 10 calories, in the case of infra-red radiation it is more than 10,000 times larger.

Because of the comparatively elementary nature of the reaction involved, the relation between the intensity $(I)$ of incident radiation and the time $(T)$ necessary to cause the 'sensation of pain' in the portion of the body exposed to radiation, is likely (in a rough way) to admit of a simple physical explanation. The problem is also connected, of course, with the determination of the tolerance limits for radiation treatment. Recently, Wolff and Hardy ${ }^{1}$, and Bigelow et $a l .{ }^{2}$, and also Drake Seager and $\mathrm{Lax}^{3}$, have described quantitative measurements on the $(T, I)$ relation: Bigelow's work relates to the forehead, and Drake Seager and Lax's to the arm. The source of radiation in the former case was an incandescent lamp and in the latter case a gas-fired furnace. The range of $I$ covered was 5-25 pyron (pyron is calories per sq. cm. per minute). It is interesting to note that the experimental results, within the limits of deviations observed, can be described by the relation

$$
T=\frac{5 \cdot 0 \times 10^{2}}{I^{2}}
$$

where $T$ is in seconds and the intensity of radiation $I$ is in pyron.

The $T-I^{2}$ relation has a simple theoretical basis. For a semi-infinite solid (initially at temperature $\theta=0$ ) which is subjected to a constant heat flux $I$ at the plane $\chi=0$, the temperature at $\chi=0$, at any time $T$, is given by

$$
\theta=\frac{2 I}{K}\left(\frac{K T}{\pi S_{p}}\right)^{1 / 2},
$$

where $K$ is the thermal conductivity of the solid, $S$ the specific heat and $p$ its density ${ }^{4}$. It is reasonable to assume that the sensation of pain is initiated when the tissue temperature rises by some fixed value $\theta_{0}$; we thus have from (2)

$$
T=\frac{\pi S \rho K}{4} \frac{\theta_{0}^{2}}{I^{2}},
$$

which is the $T-I^{2}$ law. Further, a comparison with equation (1), and taking for $(S \rho K)$ a value of about $2 \times 10^{-8}$, we have $\theta_{0} \approx 9.4^{\circ} \mathrm{C}$, , which is not unreasonable. (Since the above was written, we have seen a paper by Lloyd-Smith and Mendelssohn ${ }^{5}$. where it has been found experimentally that for the excitation of the sensation of pain a temperature rise of $15^{\circ} \mathrm{F}$. of the surface of the skin is necessary. The agreement of this result with the value of $\theta_{0}$ given above is interesting, though the rather close agreement is probably a coincidence.)

It would be of interest to determine the skin temperature simultaneously with $T-I^{2}$ measurements. It is proposed to do this, and also to extend the range of the $T-I^{2}$ relation.

$$
\text { F. C. AuLuOK }
$$

D. S. KOTHARI

University of Delhi,

Delhi.

April 25.

${ }^{1}$ Wolff, H. G., and Hardy, J. D., Phys, Rev., 27, 167 (1947).

' Bigelow, N., et al., J. Clin. Invest., 24, 503 (1945).

${ }^{3}$ Drake Seager, E. R., and Lax, C. B., Army Operational Research Group Report (declassified), No. 10/48.

"Carslaw, H. S., and Jaeger, J. C., "Conduction of Heat in Solids", 56

'Lloyd-Smith, D. L., and Mendelssohn, K., Brit. Med. J., 975 (1948).

\section{Band-Spectrum of Barium Oxide}

THE introduction of barium salts to flames or arcs leads, in presence of oxygen, to the production of a characteristic band-spectrum attributed to $\mathrm{BaO}$. Mecke and Guillery ${ }^{1}$ derived a band-head equation. Mahanti ${ }^{2}$ gave a new vibrational analysis and also made a rotational analysis. From this the spectrum was shown to arise from a ${ }^{1} \Sigma-^{1} \Sigma$ transition with $B_{e}^{\prime}=0 \cdot 30995, \quad B_{e}^{\prime \prime}=0 \cdot 36440 \mathrm{~cm}^{-1}$ and $r_{e}^{\prime \prime}=$ $1.787 \times 10^{-8} \mathrm{~cm}$. On checking Mahanti's data, several discrepancies became apparent. Thus some of the plots of $2 v_{0}$ against $J^{2},\left[2 v_{0} \simeq R(J-1)+\right.$ $\left.P(J)-2\left(B^{\prime}-B^{\prime \prime}\right) J^{2}\right]$, show anomalous changes of slope, some of the combination differences agree rather poorly, and the usual graphical method for determination of $B$, namely, by plotting $\Delta_{2} F(J)$ / $\left(J+\frac{1}{2}\right)$ against $\left(J+\frac{1}{2}\right)^{2}$, gives for $B_{2}^{\prime}$ another value than that quoted by Mahanti. These facts were disclosed by James ${ }^{3}$, who, from some relatively low-dispersion plates, was able to suggest an alternative rotational analysis with $B^{\prime \prime} \simeq 0.31, B^{\prime} \simeq 0.26$ $\mathrm{cm} \cdot{ }^{-1}$.

We therefore considered it desirable to carry out a new rotational analysis. As source we used an acetylene - air flame running at a pressure of about four atmospheres of air, fed with an aqueous solution of barium chloride. The spectrograms were taken in the first order of a $21 \mathrm{ft}$. Wood concave grating (165,000 lines) having a dispersion of about $1 \cdot 2 \mathrm{~A}$.) mm.

Eleven bands have been analysed, namely, the $5,0,4,0,3,0,3,1,2,0,2,1,1,1,1,2,0,2,0,3$ and 0,4 bands. The vibrational analysis of Mahanti has been shown to be correct; but his rotational analysis is in error. The rotational constants derived in the present work are as follows: 